\title{
Principes et protocoles en neuro-anesthésie-réanimation
}

\author{
Clément Gakuba, Franck Verdonk. Arnette Éditions, 2020, 429 pages. ISBN 978-2-7184- \\ 1514-7
}

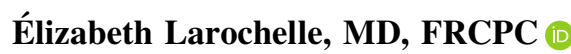

Received: 28 September 2020/Revised: 28 September 2020/Accepted: 3 October 2020/Published online: 16 October 2020

(C) Canadian Anesthesiologists' Society 2020

Le guide pratique, Principes et protocoles en neuroanesthésie-réanimation, est le résultat d'une collaboration entre deux auteurs français souhaitant synthétiser la prise en charge des patients neurolésés dans un vaste éventail de pathologies et de situations cliniques. L'ouvrage prend la forme d'un assemblage de fiches pratiques concises résumant chaque sujet de façon succincte sur deux à quatre pages. Ces fiches, classées par ordre alphabétique dans chacune des sections, permettent au lecteur une navigation très intuitive. Idéal pour une clientèle novice ou pour un expert voulant réviser les notions élémentaires, cet ouvrage est articulé en quatre chapitres : les neurosciences, l'anesthésie, la réanimation et la pédiatrie.

Le premier chapitre traite des neurosciences et détaille les connaissances fondamentales rattachées à la neuroanesthésie. Les auteurs y proposent un survol de l'anatomie adaptée, de l'imagerie diagnostique, du monitorage divers, de la pharmacologie ainsi que des principes chirurgicaux. Les tableaux et diagrammes intégrés servent à bien résumer la matière parfois complexe. En plus des notions classiques qui y sont détaillées, les auteurs consacrent une portion significative de l'ouvrage aux concepts plus modernes tels que le monitoring de la nociception, l'électro-encéphalographie ou encore les principes éthiques sous-jacents à la pratique de la neuro-anesthésie. Les dernières fiches de cette section sont consacrées aux antiplaquettaires et anticoagulants et forment un aide-mémoire pour les conduites à tenir selon qu'il s'agisse d'une urgence ou d'une chirurgie élective. Certaines notions de ce chapitre sont en décalage avec les lignes directrices canadiennes et américaines, mais

É. Larochelle, MD, FRCPC ( $\square)$

Hôpital neurologique de Montréal (CUSM), Université McGill, Montréal, QC, Canada

e-mail: elizabeth.larochelle@umontreal.ca demeurent tout de même pertinentes pour le lecteur canadien.

Le second chapitre détaille les considérations anesthésiques des pathologies vasculaires, tumorales et dégénératives les plus fréquentes ainsi que les spécificités de la chirurgie du rachis. Cette section propose un survol des conditions rattachées à la neuro-anesthésie mais pourrait laisser le lecteur sur son appétit puisque la plupart des notions n'y sont qu'effleurées. Les auteurs proposent une série de protocoles simplifiés et illustrés qui facilitent la compréhension. Les références sont toujours mises en évidence, et proviennent généralement d'institutions reconnues. Chaque fiche est détaillée de façon similaire: généralités/physiologie, clinique/imagerie, prise en charge chirurgicale, préopératoire, conditionnement, stratégie anesthésique et complications. Si certaines de ces sections sont riches en informations pertinentes et très utiles pour l'anesthésiologiste, d'autres sont parfois redondantes (ex. antibioprophylaxie, voies veineuses, préopératoire, post-opératoire). Par ailleurs, plusieurs encadrés de couleur différente offrent un résumé de faits saillants pouvant guider le lecteur.

Le troisième chapitre est consacré à la réanimation en lien avec la neuro-anesthésie. Les auteurs adressent autant les complications plus fréquentes telles que le diabète insipide et l'hypertension intracrânienne que les complications plus rares telles que les thrombophlébites crâniennes. Les facteurs de risque et principes de prise en charge sont détaillés. Le lecteur retrouvera plusieurs organigrammes décisionnels à l'intérieur desquels le niveau de détail et la rigueur y sont appréciables. Les prises en charge suggérées demeurent sommaires, mais amplement suffisantes pour initier un traitement ciblé.

Le dernier chapitre du livre traite de la pédiatrie. Les notions abordées font référence aux pathologies et principes propres à l'anesthésie pédiatrique. Les auteurs réussissent à éviter les répétitions des sujets déjà abordés 
dans la section adulte. Cette section ne se prête pas à tous puisque la pratique pédiatrique est souvent séparée des centres hospitaliers adultes. Elle demeure néanmoins un aide-mémoire pertinent.

En résumé, la force de cet ouvrage, qui est le premier guide pratique de neuro-anesthésie à être publié en français, réside dans sa capacité à simplifier, synthétiser et illustrer les notions complexes de neuro-anesthésie. Le lecteur s'y retrouvera très facilement grâce à l'approche par thème et pourra creuser davantage les sujets grâce aux nombreuses références provenant d'institutions réputées. En revanche, cet ouvrage n'est pas conçu pour en faire une lecture intégrale et n'est donc pas idéal pour apprivoiser la matière en détail. En respectant ce pourquoi cet ouvrage a été conçu, autant le lecteur avisé qui souhaite avoir accès à une aide cognitive spécifique rapidement que le lecteur moins aguerri qui souhaite accéder à une information de qualité bien synthétisée y trouveront leur compte.

\section{Conflits d'intérêts Aucun.}

Source de financement Aucun.

Responsabilité éditoriale Cet article a été traité par Dr Étienne de Médicis, rédacteur de la langue française, Journal canadien d'anesthésie.

Publisher's Note Springer Nature remains neutral with regard to jurisdictional claims in published maps and institutional affiliations. 